arrangements and self-reported suicidality but not suicidality as indicated by administrative records. A limitation is that selective participation may bias the results for self-reported suicidality; however, the large sample size enabled participants to be followed up using death records, which are less vulnerable to bias.

\section{OP64 IMPACT OF POVERTY TRAJECTORIES ON CHILDREN'S HEALTH AND MATERNAL MENTAL HEALTH: EVIDENCE FROM THE UK MILLENNIUM COHORT STUDY}

S Wickham, ETC Lai*, B Barr, DC Taylor-Robinson. Department of Public Health and Policy, University of Liverpool, Liverpool, Liverpool, UK

\subsection{6/jech-2018-SSMabstracts.63}

Background The number of children living in poverty in the United Kingdom (UK) is rising. Child poverty has been shown to cause poor mental and physical health outcomes that last into adulthood. Poverty also puts families in distress. The aim of the study was to understand the prevalence of different poverty trajectories for UK children, and their associations with multiple child health and maternal mental health outcomes.

Methods We analysed data on 11565 children who participated in sweeps of the UK Millennium Cohort Study from ages 9 months to 14 years. Outcomes were: (i) mental health at age 14, measured by the Strengths and Difficulties Questionnaire (SDQ); (ii) physical health at age 14, measured by overweight/obese and any longstanding illness; and (iii) maternal mental health, measured by Kessler 6 scale. The main exposure of interest was relative poverty (less than 60\% of median of equivalised household income). Poverty trajectories measured at 9 months, 3, 5, 7, 11 and 14 years were characterised using latent class analysis. Odds ratios (ORs) and 95\% confidence intervals (CIs) were estimated using multivariable logistic regression models, adjusted for child sex, maternal education and maternal ethnicity.

Results Four poverty trajectories were identified: class 1 (61.2\% of children) (stable never-poor, reference group), class $2(14.2 \%)$ (poverty in early childhood), class $3(5.2 \%)$ (poverty in late childhood) and class 4 (19.4\%) (persistent poverty). Any exposure to poverty was associated with increased risk of longstanding illness, and worse mental health outcomes for both mothers and children, with the largest effects for persistent poverty. Compared with children who were never poor, those from persistently poor households were at a higher risk of having mental health problems [SDQ score $\geq 17$ (aOR: 2.76; 95\% CI 2.29 to 3.34)], physical health problems [being overweight/obese (aOR: 1.23 ; 95\% CI 1.07 to 1.41); longstanding illness (aOR: 1.82, 95\% CI 1.55 to 2.15)] and mothers under psychological distress [Kessler 6 scale score $\geq 6$ (aOR: 2.60; 95\% CI 2.26 to 3.00)].

Conclusion Persistent poverty affects one in five UK children and is associated with negative impacts on child and maternal health, particularly mental health. Any exposures to poverty mainly in early or late childhood were also associated with worse outcomes. One of the limitations of this study is that household income was self-reported. Policies that reduce child poverty and its consequences are likely to improve health across the life course.
OP65 MENTAL HEALTH COMPETENCE AT AGE 11 AND ITS ASSOCIATION WITH RISK-TAKING HEALTH BEHAVIOURS AT AGE 14: FINDINGS FROM THE UK MILLENNIUM COHORT STUDY

${ }^{1} \mathrm{~S}$ Hope ${ }^{*},{ }^{1} \mathrm{E}$ Rougeaux, ${ }^{2} \mathrm{~J}$ Deighton, ${ }^{1} \mathrm{R}$ Viner, ${ }^{1} \mathrm{C}$ Law, ${ }^{1,3} \mathrm{~A}$ Pearce. ${ }^{1}$ University College London Great Ormond Street Institute of Child Health, University College London, London, UK; ${ }^{2}$ Evidence Based Practice Unit, Anna Freud National Centre for Children and Families, London, UK; ${ }^{3}$ MRC/CSO Social and Public Health Sciences Unit, University of Glasgow, Glasgow, UK

\subsection{6/jech-2018-SSMabstracts.64}

Background Risk-taking health behaviours initiated during adolescence can track into adulthood and influence lifelong health and social outcomes. Promoting positive mental health, particularly through enhancing competencies (such as pro-social skills), may help prevent the development of risk-taking behaviours and thus support future health. We investigated the relationship between skills-based components of positive mental health in childhood (Mental Health Competence, MHC) and teenage risk-taking behaviours, using data from a UK-representative population cohort, the Millennium Cohort Study ( 18000 children born 2000-2002).

Methods Cohort members (CMs) reported on a number of common risk-taking behaviours at 14 years(y), categorised as follows: ever tried cigarettes (yes/no), ever tried e-cigarettes (yes/no), alcohol consumption (never tried, tried binge-drinking $[5+$ alcoholic drinks at a time], tried but never bingedrinking), ever tried illegal drugs (yes/no), ever engaged in antisocial behaviour (yes/no, any of: theft, graffiti, public property damage, carrying a weapon, using a weapon, breaking and entering), and sexual contact with another young person (yes/no). A four-class latent measure of MHC summarised learning skills and prosocial behaviours at $11 \mathrm{y}$ (maternal report): High, High-Moderate, Moderate, Low. We used logistic regression to estimate odds ratios (OR) for binary outcomes and multinomial regression to estimate relative risk ratios (RRR) for categorical outcomes, adjusting for confounding by socio-demographic characteristics, maternal mental health and alcohol consumption, parenting at $3 \mathrm{y}$, and puberty reported at $11 \mathrm{y}$. Sample design and attrition were accounted for with weights and item missingness with multiple imputation.

Results $17 \%$ of CMs had ever tried smoking cigarettes and $18 \%$ had ever tried e-cigarettes. $49 \%$ of $\mathrm{CMs}$ had consumed alcohol, including 11\% binge-drinking. Trying illegal drugs, displaying anti-social behaviours and having sexual contact were less prevalent (6\%-10\%). Compared to CMs with High MHC, those with Low, Moderate, or High-Moderate MHC at age 11 were more likely to have taken part in risk-taking behaviours. After adjustment for potential confounding, elevated risks remained for Low $\mathrm{MHC}$ in relation to binge drinking (RRR: 1.6 [95\% CI 1.1 to 2.3]), having tried smoking cigarettes (OR: 2.1 [1.5-2.9]), e-cigarettes (OR: 1.4 [1.02.0], illegal drugs (OR: 1.9 [1.3-2.9]), and anti-social behaviour (OR: 1.8 [1.2-2.6]) but not sexual contact (OR: 1.1 [0.7-1.6]).

Conclusion MHC at the end of childhood was associated with risk-taking behaviours in mid-adolescence in a representative UK cohort. Interventions that improve MHC skills in childhood may help reduce risk-taking behaviours at this crucial stage in the lifecourse, improving wellbeing in adolescence and into adulthood. 